\title{
The influence of bars in the star formation history and chemical evolution of disk galaxies
}

\author{
Patricia Sánchez-Blázquez ${ }^{1}$ \\ ${ }^{1}$ Instituto de Astrofísica de Canarias, \\ c/Via Lactea S/N, E38205, La Laguna (Tenerife), Spain \\ email: psanchezb@iac.es
}

\begin{abstract}
This is a pilot study part of a larger program devoted to understand the influence of bars in the evolution of galaxy disks. Some of the goals of this observational program are to compare the stellar metallicity gradients of galaxies with and without bars, the role of bars in the redistribution of mass and angular momentum and to study the evolution of the metallicity gradients with time. Some promising results are presented as well as some words of caution.
\end{abstract}

Keywords. galaxies: stellar content, galaxies: spiral, galaxies: abundances

\section{Introduction}

During disk assembly, secular evolution must have played a role in shaping the structure of disk galaxies as we see them at $\mathrm{z}=0$. Non-axisymmetric instabilities, particularly bars, drive a substantial redistribution of mass and angular momentum in the disk (e.g., Debattista et al. 2006). Since bars appear to strongly affect the overall dynamics and evolution of galaxies, it is reasonable to suspect that bars can be responsible for significant chemical evolution as well, since mixing by global gas flows will clearly change abundance profiles in the disk (see Friedli 1998; Roskar et al. 2008; Sánchez-Blázquez et al. 2009). While there have been several studies finding that barred galaxies have a shallower gasphase metallicity gradient than non-barred ones (e.g., Pagel \& Edmunds 1981; VilaCostas \& Edmunds 1992; Martin \& Roy 1994), an equivalent study in the stellar phase metallicities remain to be done. In this paper, we present a pilot study devoted to analyse the techniques to show the viability of the study.

\section{Data}

All observations were taken with the Gemini South Multi-Object Spectrograph (GMOS). Long slit spectroscopy along the major axes of the galaxies was performed with an slit width of 0.75 " and with the B600-G5303 grating. We performed a binning of $4 \times 2$ giving to an spectral resolution of FWHM $=3.5 \AA$ and an spatial scale of 0.292 $\operatorname{arcsec} /$ pixel. Our spectra covers the wavelength range from 4050-6750 $\AA$.

\section{Star Formation Histories}

We use here STECKMAP (Ocvirk et al. 2006a,b), which is a method that, simultaneously, recover the kinematic and stellar population properties via a maximum a posteriori algorithm. The method is not parametric and does not make any a priori assumption about the star formation history. The only condition that STECKMAP impose is that 

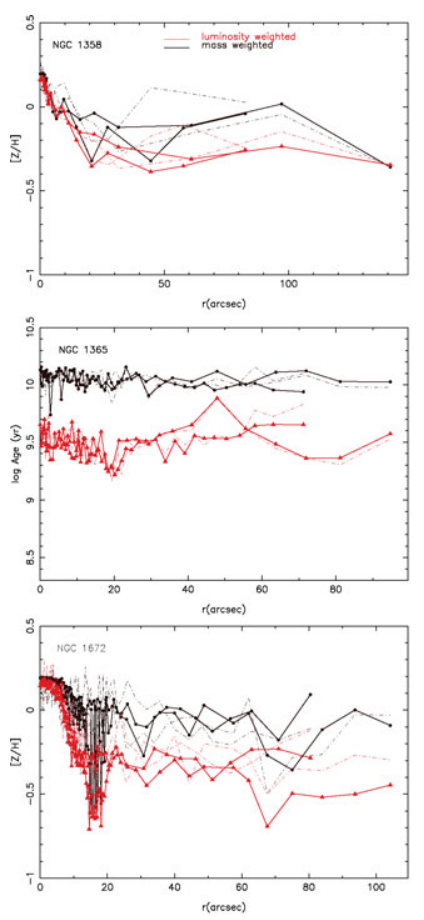
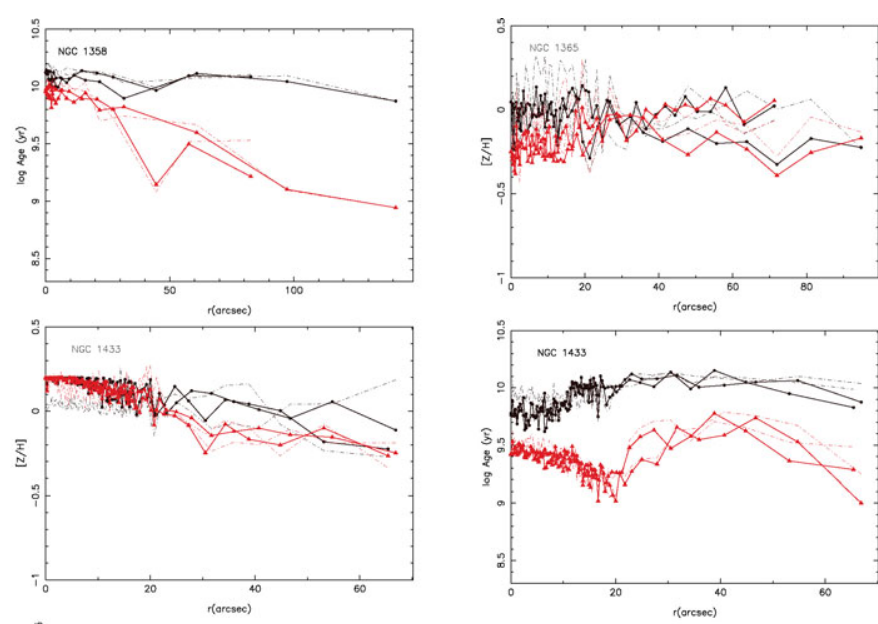

Figure 1. Mass- (black lines) and luminosity-weighted (red lines) age and metallicity gradients derived from the recovered star formation history. Solid lines indicates the results using V09 models while dotted-dashed lines those obtained with BC03.

the different unknowns (spectral age distribution, age-metallicity relation, line-of-sight velocity distribution) change smoothly with time. This constrain avoid spurious burst of star formation that appear in other methods. For this work, we use a Laplacian smoothing Kernel (see Ocvirk et al. 2006a for the different possibilities). We use the new stellar population models by Vazdekis et al. (2009, V09 hereafter) that include a new and improved stellar library MILES (Sánchez-Blázquez et al. 2006; Cenarro et al. 2007) but we also check that our results do not depend on the chosen model by repeating the analysis using Bruzual \& Charlot (2003, BC03 hereafter) models. We can use the derived star formation histories to obtain luminosity- and mass-weighted age and metallicity gradients. Figure 1 shows the results. Below, we analyse the galaxies individually.

- NGC 1358: is a Seyfert galaxy with a bar, and morphologically classified as an S0. The luminosity-weighted age gradient is very steep, without a transition between the bulge and the disk region, reflecting again, the inside-out formation of this galaxy. However, as in the NGC 1433, the mass weighted age gradient is very flat and the absolute value of the age is high $(\sim 10 \mathrm{Gyr})$, indicating that the whole disk is dominated in mass by old stellar populations. The metallicity gradients in the bulge region (both, massweighted and luminosity-weighted) are very steep, as commonly found in spheroids, but it flattens in the disk region, where a significant gradient is not found.

- NGC 1365: is a (R')SBb(s)b galaxy. The luminosity weighted age profile shows a minimum at the position of the circumnuclear ring but it is very flat otherwise. The massweighted age profile is compatible with being flat all across the galaxy. The metallicity gradient is also compatible with zero all ac-cross the galaxy (although in one side of the galaxy the lines could be indicating a slope of $\sim 0.05 \mathrm{dex} / \mathrm{kpc}$, the result is not so clear if $\mathrm{BC} 03$ models are used). 
- NGC 1433: is a ringed barred spiral of revised de Vaucouleurs type (R')SB(r)ab. The nuclear ring is a small blue feature at 19" in angular diameter. The luminosity-weighted age gradient of this galaxy show a minimum at the ring position, reflecting a younger stellar population in this structure. In the external parts, the age gradient show a negative slope reflecting the inside-out formation of this galaxy. In the mass-weighted age profile, however, the minimum at the ring value and the negative slope are not present, indicating that the majority of the stellar mass is composed of old stellar populations ( 10 Gyr). The bulge of the galaxy may have a larger contribution of a younger component than the disk, reflected in its lower mean mass-weighted age ( $\sim 5 \mathrm{Gyr})$. The overall metallicity gradient is quite steep, due mainly to the metallicity difference between the bulge and disk region.

- NGC 1672 This galaxy is an Sbc(s) and is the galaxy with the later morphological type of our sample. This galaxy have a circumnuclear ring of star formation located on an inner Lindblad resonance (Díaz et al. 1999) surrounded by a nucleus with lowlevel activity (Verón-Cetty \& Verón 1986). The circumnuclear ring of star formation can be seen as a deep in the radial luminosity-weighted age distribution. However, it does not represent an important fraction of the mass as it does not appear in the massweighted distribution. While the mass-weighted age gradient in the disk region is flat, the luminosity weighted show a negative slope, indicating an inside-out formation of the disk. The metallicity gradient is very flat in the disk region but very steep in the bulge.

In general, the results obtained are very similar using either V09 or BC03. Furthermore, the profiles in both sides of the galaxy are very similar, giving confidence in our results. Something that can be seen in all the figures is that the luminosity weighted metallicity gradient tend to be smaller than the mass-weighted one. This, in principle, indicates that the younger populations (weighting more in the luminosity than in the mass-weighted means) are more metal poor than the old ones. This is contrary to our expectations due to the chemical evolution enrichment inside the galaxies and we suspect that this is an artifact of the method. Therefore, we tested our results to the use of different wavelength ranges and different regularization of all the parameters. To do this, we built two different star formation histories, one exponential and another one constant using the V09 models. We impose an age metallicity relation where the metallicity increase linearly with time. Then, we recovered the star formation histories and mass-metallicity relation using different wavelength ranges and regularizations. We performed the experiment with both models, V09 and B03. To quantify the success of the solution recovery we compute the mass- and luminosity weighted age and metallicities in both, the synthetic and the recovered star formation histories. Figure 2 shows the comparison. As can be seen, while the mass weighted values are usually well-recovered, independently of the wavelength range, regularization or model used, the recovery of the luminosity weighted values depends on the star formation history. While the luminosity weighted metallicity is very well recovered in the case of a constant star formation history, the recovered metallicity is systematically lower when an exponential star formation history is used. The luminosity weighted age also tend to be lower than the real one in both cases, although the differences are not very large. Therefore, we think that the lower values of the mean luminosity-weighted metallicity than the mass-weighted metallicity can be due to an artifact of the method and we will not try to interpret it.

\section{Summary}

We are carrying on a project to understand the star formation history of disk galaxies and, in particular, the influence of the bar in producing large scale mixing. In this paper 

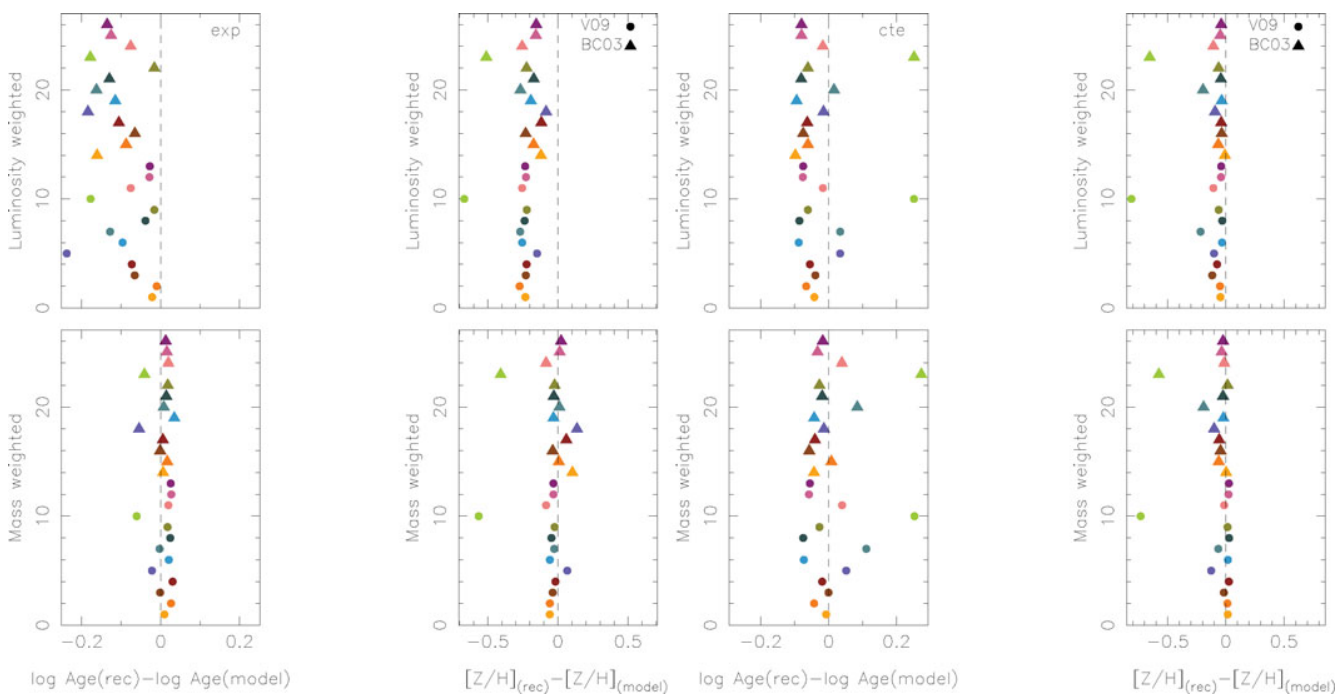

Figure 2. Differences between the model and the derived mean age and metallicity- weighted with age and mass- obtained using different wavelength ranges and regularizations for an exponential (exp in the inset) and a constant star formation history (cte in the inset). Different colors indicate the results obtained in different wavelength ranges and using different regularizations for the solution. Filled circles represent the results obtained with V09 while triangles those using $\mathrm{BC} 03$.

we have tested the techniques we are planning to use to derive star formation histories and chemical evolution of disk galaxies using long-slit spectroscopy. In the four analysed galaxies we have found a variety of metallicity gradients, a flat mass-weighted age gradient and a mild negative luminosity weighted age gradient, consistent with an inside-out formation of the discs. Similar metallicity gradients have been found in external galaxies without bars too (Yoachim \& Dalcanton 2008; MacArthur et al. 2009). In the future, we plan to compare these gradients with the gas-phase abundances and try to build a consistent picture of the star formation history at different radius.

\section{References}

Bruzual, G. \& Charlot, S. 2003, MNRAS, 344, 1000

Cenarro, A. J. et al. 2007, MNRAS, 374, 664

Debattista, V. D., Mayer, L., Carollo, M., Moore, B., Wadsley, J., \& Quinn, T. 2006, ApJ, 645, 209

Díaz, R., Carranza, G., Dottori, H., \& Goldes, G. 1999, ApJ, 512, 623

Friedli, D. 1998 ASPC, 147, 287

MacArthur, L., González, J. J., \& Courteau, S. 2009, MNRAS, 395, 28

Martin, P. \& Jean-Rene, R. 1994, ApJ, 424, 599

Ocvirk, P., Pichon, C., Lancon, A., \& Thiébaut, E. 2006, MNRAS, 365, 46

Ocvirk, P., Pichon, C., Lancon, A., \& Thiébaut, E. 2006, MNRAS, 365, 74

Pagel, B. \& Edmunds, M. ARA $\& A$ A, 19,77

Roskar, R., Debattista, V. P., Quinn, T. R., Stinson, G. S., \& Wadsley, J. 2008, ApJ, 648, 79

Sánchez-Blázquez P. et al. 2006, MNRAS, 371, 703

Sánchez-Blázquez, P., Courty, S., Gibson, B. K., \& Brook, C. B. 2009, MNRAS, 398, 591

Veron-Cetty, M.-P. \& Veron, P. 1986, A\& A, 160,39

Vila-Costas, M. B. \& Edmunds, M. G. 1992, MNRAS, 259, 121

Yoachim, P. \& Dalcanton, J. J. 2008, ApJ, 683, 707 